Obgleich die Übereinstimmung der $\mathrm{Re}$ sultate unter sich, bei wiederholter Ausführung der Arbeit eine gute zu nennen war, aber doch mit denen durch Magnesiafällung erhaltenen bisweilen sehr von der Richtigkeit abwichen, so wird doch vielleicht durch diese Versuche Veranlassung gegeben, die Ursache dieser Fehler zu erforschen und zu beseitigen. Die directe Bestimmung als dreibasisch phosphorsaur. Kalk würde dann einfacher und schneller auszuführen sein als diejenige mit Anwendung von Chlormagnesiumlösung; auch würde der dreibasisch phosphorsaure Kalk wegen seines höhereu Atomgewichts gegenüber der pyrophosphorsauren Magnesia bei der quantitativen Bestimmung der Vorzug zu geben sein.

Magdeburg, Juni 1888.

\section{Über den Gebrauch der Knochenkohle zur Entfärbung dunkler Znckerlösungen.}

\section{Von \\ E. Bauer.}

So eingehende Arbeiten über die Absorption von Zucker, Salzen, Farbstoffen u. dgl. durch Knochenkohle vorliegen, wie jene von Bodenbender, Ventzke, Scheibler, Walberg, Graham, Walkhoff u. A., so wenig Klarheit und Gleichmässigkeit herrscht beim Gebrauche des Spodiums zur Entfärbung dunkler Zuckersäfte behufs Polarisation. Die hierüber in der Literatur zu findenden Angaben, ebenso wie die unter den practischen Chemikern gebräuchlichen Gepflogenheiten weichen ungemein von einander ab. So finden wir über die Menge und Beschaffenheit des zu verwendenden Spodiums, über Dauer der Einwirkung und Correction des zu begehenden Fehlers entweder gar keine oder höchst unvollkommene Angaben. Meist wird nur im Allgemeinen angerathen, bei dunklen Nachproducten, welche durch Bleiessig nicht genügend entfärbt werden, etwas bei $130^{\circ}$ getrocknete Knochenkohle zu verwenden. Über einen in Rechnung zu ziehenden Absorptionsfactor wird nichts bemerkt. Oder wie $L_{a}$ andolt empfiehlt, werden 30 bis 40 ee vom Bleiessigfiltrat mit 3 bis $6 \mathrm{~g}$ gepulverter, scharf getrockneter Knochenkohle zusammengebracht, anhaltend geschüttelt und 12 bis 24 Stunden stehen gelassen. Das Absorptionsvermögen der Kohle soll für reine Zuckerlösungen vorher bestimmt und entsprechend zu berücksichtigen sein.
Abgesehen davon, dass eine 12 bis 24 Stunden dauernde Einwirkung zur Entfärbung der dunkelsten Producte völlig unnöthig erscheint, ist es durchaus nicht gleichgiltig, ob die Absorption eine Minute oder ein beliebig Vielfaches derselben dauert, ebensowenig wie es dasselbe ist, ob eine reine Zuckerlösung oder derartig mannigfaltige Gemenge vorliegen, wie Melasse, Osmosewässer oder Entzuckerungslaugen, die neben wechselnden Mengen von Zucker Salze und colloide Substanzen verschiedenster Art enthalten. Auch Frühling und Schulz verfahren in ähnlicher, wenn auch etwas strengerer Weise, lassen jedoch den letzteren Umstand auch unberücksichtigt.

Nun gibt es allerdings Kohle, deren Absorptionsvermögen gering ist, so dass der zu machende Fehler unbedeutend ist; ja, es sei hier eines Falles Erwähnung gethau, dass eine Melasse nach der Behandlung mit Spodium, deren Absorptionsvermögen genau bestimmt wurde, sogar eine um 0,3 Proc. höhere Polarisation zeigte, ein Umstand, der selbstredend auf die Verminderung einer linksdrehenden Substanz zurückzuführen ist. Bei Kohle jedoch, deren Absorptionsvermögen ein kräftiges, wie dies beispielsweise bei durch Salzsäure gereinigter immer der Fall ist, werden die Fehler bei Annabme eines bestimmten Absorptionsfactors bei wechselnden Zuckermengen und verschiedenen Producten beträchtlich schwanken.

Eine dem Handel entstammende, sog. chemisch reine, mit Salzsäure gereinigte Koble, welche ein auffallend hohes Absorptionsvermögen besass, gab Veranlassung zu folgenden Versuchen.

Vorerst wurde das Absorptionsvermögen für reinen Zucker geprüft. Als solcher dieute ein aus Alkohol mehrmals umkrystallisirter Zucker, welcher alkoholfeucht aufbewalirt, jedoch vor jedem Versuche bei $95^{\circ}$ getrocknet wurde. Die Knochenkohle wurde zum Theil pulverförmig, zum Theil in Griesform verweudet. Vor dem Gebrauche wurde dieselbe einem auf $130^{\circ}$ erhitzten Luftbade entnommen.

Ein kalter entwässerter Auszug zeigte keinerlei Reaction auf äusserst empfindliches Lackmuspapier. Es wurde eine Probe von $8,959 \mathrm{~g}$ mit Wasser ausgek ocht und filtrirt. Das Filtrat mit Barytlösung und Rosolsäure titrirt, ergab einen Gehalt von 0,065 Proc. Säure auf Salzsäure berechnet. Da zu den Absorptionsversuchen immer $5 \mathrm{~g}$ Kohle und 50 cc Lösung verwendet wurden, so würde sich hieraus die Menge der freien Säure auf $3 \mathrm{mg}$ oder etwa 0,006 Proc. berechnen, eine Menge, der eine invertirende Kraft nicht 
beizumessen ist. Zur Polarisation diente ein Soleil-Ventzke'scher Farbenapparat. Der Durchschnitt aus 6 Ablesungen wurde als geltend angenommen.

I. Absorption reiner Zuckerlösung. Zur Fesstellung derselben wurden folgende Versuche ausgeführt:

Verwendet $21,519 \mathrm{~g}$, welche in $200 \mathrm{cc}$ gelöst wurden.

Ablenkung im $200 \mathrm{~mm}$ Rolı 40,73, daher 98,46 Proc. 5 g pulverförmige Knochenkohle genau 2 Min. lang auf $50 \mathrm{cc}$ obiger Lösung unter kräftigem Schütteln einwirken gelassen und rasch durch ein trockenes Filter filtrirt.

Ablenkung - $\quad 38,66^{\circ}$, daher 93,46 Proc.

Bei $10,7745 \mathrm{~g} \mathrm{Zucker} \mathrm{wurden} \mathrm{also} \mathrm{5,07} \mathrm{Proc.}$ absorbirt.

Ebenso wurde mit griesförmiger Knochenkohle verfahren.

Ablenkung - $38,6^{\circ}$, daher 93,3 Proc. oder vom Zucker 5,21 Proc. absorbirt.

Dass hier bei dem gröberen Spodium, entgegen den sonstigen Thatsachen, die Wirkung eine bedeutendere war, ist offenbar nur einem zufälligen Umstande, und zwar höchstwahrscheinlich einem stärkeren Schütteln zuzuschreiben.

Ein zweiter Versuch mit derselben Lösung und derselben Knochenkohle, jedoch weniger geschüttelt ergab eine Ablenkung

ron $39,15^{\circ}$,

daher 94,6 Proc.

Also ein geringer Unterschied in der mechanischen Behandlung ergab sogleich einen Polarisationsunterschied von 1,3 Proc.

Einfluss des Salzgehaltes auf die Zuckerabsorption. Wie weit ein Salzgehalt, im Verhältniss der in der Melasse vorkommenden Salze die Zuckerabsorption zu beeinflussen vermag, sollte folgender Versuch ergeben.

Verwendet $26,6866 \mathrm{~g}$ Zucker zu 200 ce gelöst. Hiervon $100 \mathrm{ec}$ mit $2,39 \mathrm{~g}$ löslichen Schlempekohlensalzen versetzt, entsprechend einem Salzgehalt der Melasse von 9 Proc. Directe Polarisation ohne Knochenkohle $49,2^{\circ}=96,04$ Proc. Mit $5 \mathrm{~g}$ gepulverter Knochenkohle auf 50 ce Lösung bei einer 2 Minuten währenden Einwirkung unter kräftigem Schütteln :

Polarisation $46,3^{\circ}$,

daher 90,38 Proc.

Die Absorption betrug daher in diesem Falle bei 12,81.49 g 5,89 Proe. vom 'Zucker.

Nicht nur, dass in diesem Versuche eine grössere Zuckermenge in Verwendung kam, nach Walberg's Versuchen (Z. Zucker. 1874 S. 861) aber die procentische Absorption mit der Concentration abnimmt, wurde hier trotz des Salzgehaltes eine noch stärkere Absorption beobachtet, ein Umstand, der einestheils daranf hinweist, dass ein Salzgehalt in diesem Verbältniss die Absorption nicht beeinträchtigt, dass aber auch bei möglichster Einlıaltung gleicher Versuchsbedingungen Schwan- kungen in der Absorption nicht zu vermeiden sind.

Versuche mit völlig neutraler Knochenkohle. Wenngleich nun, wie betont, der geringen Säurespur des Spodiums unmöglich eine beeinflussende Wirkung zuzuschreiben ist, wurde für die folgenden Versuche dasselbe einer weiteren Reinigung durch Waschen mit Ammoniak, heissem Wasser und 10 stündigem Trocknen bei $130^{\circ}$ unterworfen. Wie jedoch weiterhin ersichtlich, ist ein Unterschied in der absorbirenden Kraft derart behandelter Knochenkohle nicht wahrnehmbar.

Angewendet: $23,821 \mathrm{~g}$ in $200 \mathrm{cc}$ Lösung.

Directe Polarisation 97,67 Proc.

Unter Anwendung von $5 \mathrm{~g}$ Knochenkohle auf $50 \mathrm{cc}$ Lösung, 2 Minuten Daner und kräftigem Schütteln: Polarisation 92,02 Proc. Von $11,632 \mathrm{~g}$ Zucker wurden also 5,8 Proc. absorbirt.

Einfluss der Zeitdauer auf die Absorption. Um die durch ungleichmässiges Schütteln hervorgebrachten Differenzen zu vermeiden, wurde bei nachfolgenden Versuchen, welche im Übrigen genau den früheren angepasst waren, die Flüssigkeit zu der im Kölbchen befindlichen Kohle geschüttet, aber ruhig stehen gelassen.

Angewendet: $26,048 \mathrm{~g} \mathrm{zu} 200 \mathrm{cc}$ gelöst. Directe Polarisation ohne Knochenkohle 97,31 Proc. Mit $5 \mathrm{~g}$ pulvf. Spodium und

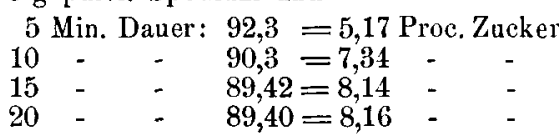

Die Absorption war also, wie ersichtlich, in 20 Minuten beendet, wobei eine Polarisationsdifferenz von 7,94 Proc. erreicht wurde.

Absorption in salzsaurer Lösung. Da es von Interesse schien, die Absorptionsfähigkeit der Knochenkoble in salzsaurer Lösung kennen zu lernen, unter Umständen, wie sie genau den für Clerget'sche oder Creydt'sche Inversionsmethode angegebenen Bedingungen entspricht, wurden weiterhin noch einige diesbezügliche Versuche angestellt.

Abgewogen: $22.2624 \mathrm{~g}$ 7ucker, in 200 ce gelöst. Directe Polarisation $41,63^{\circ}=97,4$ Proc. Zucker. $50 \mathrm{ec}$ hiervon wurden genau nach den Angaben von Reichardt u. Bittmann (Z. Zucker. 1882 S 764) der Inversion unterworfen. Die verwendete Süure hatte ein sp. Gew. von 1,188 bei $15^{\circ}=38$ Proc. $\mathrm{H} \mathrm{Cl}$.

Zur Entfärbung der concentrirten Lösung wurden $5 \mathrm{~g}$ gepulverte Knochenkohle in der Dauer von 2 Minuten einwirken gelassen ${ }^{1}$ ). Die doppelt ver-

1) Die von Reichardt und Bittmann angegebene Menge von 0,5 bis $1 \mathrm{~g}$ genügen nicht, um dunkle Melassenlüsungen für den Farbenapparat zu entfärben. 
dünnte Lösung polarisirte bei $22,5^{\circ}$ ohne Spodium $-6,83^{\circ}$.

Hieraus berechnet sich die Drẹungsdifferenz $\mathrm{S}$, wenn die ursprüngliche Drehung für das $200 \mathrm{~mm}$ Rohr $41,63^{\circ}$ betrug:

$$
\begin{aligned}
& \mathrm{A}= 41,63.2=+83,26 \\
& \mathrm{~B}=-6,83.4=-27,32 \\
& \hline \mathrm{S}=110,58
\end{aligned}
$$

Mit Zuhilfenahme der Tuchschmidschen Tabelle für die Temperatur von $22,5^{\circ}$ ergab sich ein Gehalt von 97,35 Proc. Zucker. Wie ersichtlich stimmt das Resultat fast genau überein mit der ursprünglich durch directe Polarisation gefundenen Menge.

Mit Spodinm

$$
\begin{aligned}
& \text { Drehung }=-6,78 \\
& \text { daher } \mathrm{A}=-6,78.4=-27,12 \\
& \mathrm{~B}=41,63.2=83,26 \\
& \mathrm{~S}=\mathbf{1 1 0 , 3 8}
\end{aligned}
$$

Dieser Zahl entspräche nach Tuchschmid's Tabelle ein Gehalt von 97,29 Proc. Zucker $^{2}$ ).

Die durch die Knochenkohle bewirkte Absorption betrug also $0,05^{\circ}$ der Skala, während der wahre Zuckerwerth um 0,11 Proc. herabgedrückt wurde. Die Absorption in stark salzsaurer Lösung ist gegenüber einer neutralen eine verschwindend kleine.

Der Inversionsversuch wurde wiederholt, nur dass statt der pulverförmigen Knochenkoble eine solche von Griesform verwendet wurde.

Abgewogen: $16,1304 \mathrm{~g} \mathrm{zu} 100 \mathrm{cc}$ gelöst.

Directe Polarisation 98,68 Proc.

Drehung der invertirten Lösung bei $25^{\circ}$

ohne Spodium - 9,45 mit Spodium - 9,37

Die sich lieraus nach Tuchschmid's Tabelle ergebenden Werthe entsprechen:

$$
\text { ohne Spodium }=98,23
$$$$
\text { mit } \text { Spodium }=98,03
$$

Differenz $\quad 0,2$

Das in diesem Falle geringe Übereinstimmen der ursprünglichen Polarisation mit der nach der Inversionsmethode gefundenen ist der hohen Beobachtungstemperatur von $25^{0}$ zuzuschreiben.

Die Absorptionsdifferenz war also abermals eine geringe, wenn auch, wahrscheinlich in Folge des Temperaturunterschiedes, eine höhere, als im ersten Versuche ${ }^{3}$ ).

2) Wird zur Berechnung die neue Landolt'sche Formel zu Grunde gelegt (S. 132 d. Z),

$$
\mathrm{R}=\frac{100 \cdot \mathrm{S}}{142,4-0,5 \mathrm{t}} \text {, }
$$

so erhält man gegenüber den vorliegenden Resultaten und der ursprünglichen Polarisation zu hohe Resultate, nämlich

ohne Spodium 98,64 Proc. gegen 97,35

$$
\text { mit Spodium 98,48 }
$$

$$
\text { Differenz } \overline{0,16}
$$

3) Hier ergeben sich auf Grund der Landolt' schen Formel:
Jedenfalls ist ersichtlich, dass die Anwendung von Knochenkohle bei der Inversionsmethode einen nur sehr geringen Fehler bewirlst.

Einfluss der mit Spodiu!m behandelten Zuckerlösung auf Soldainisches Reagens. Die auffallend scheinende $\mathrm{Ab}$ sorptionskraft der Kohle liess die Erwägung nicht so fern liegen, dass dieselbe eben eine nur scheinbare, vielmehr eine theilweise Hydratisation, veranlasst durch die Anziehung der Poren, stattfinden könne, die nach der Spodiumbehandlung erfolgende Minusdrehung also auf die Bildung linksdrehenden Invertzuckers zurückzuführen wäre. Die geringe Absorption in der invertirten Lösung schien für diese Annahme zu sprechen.

Die chemische Wirkungsfähigkeit der Knochenkohle auf viele Stoffe ist ja bekannt; es sei hier nur auf die Spaltung der Indigoschwefelsäure, des Jodkaliums, die Zersetzung der schweren Metallsalze, so wie auf die unläugbare chemische Wirkung bei der Knochenkohlefiltration des Alkohols hingewiesen.

Anderseits ist, wie zuerst von Fensky beobachtet, später von Soubeyran, Jodin, Duvin, Horsin-Déon u. A. bestätigt wurde, die Inversion des Rohrzuckers durch blosses Kochen mit Wasser eine unzweifelhafte Thatsache (siehe Lippmann "Die Zuckerarten", S. 124). Es konnte also immerhin möglich sein, dass die in der Capillare vor sich gehende Verdichtung des Wassermoleküls unter gleichzeitiger Mitwirkung der Anziehung der 'Lellwände eine Inversionserscheinung hervorbringen könne.

Gewogen: $23,821 \mathrm{~g}$ Zucker anf 200 cc gelöst. Directe Polarisation $97,67^{\circ}$ $50 \mathrm{ce}$ der Lösung wurden mit $5 \mathrm{~g}$ griesförmiger Knochenkohle 2 Minuten lang unter krüftigem Schütteln digerirt und unmittelbar filtrirt.

Polarisation

$92,02^{\circ}$

Zur Prüfung der Reductionsfähigkeit wurde eine genau nach Bodenbender (Z. Zucker. Febr. 1887) hergestellte Kupferlösung verwendet. $100 \mathrm{cc}$ derselben wurden vorerst für sich allein nach Angabe Bodenbender's 8 Min. über einem Asbestring und freier Flamme gekocht, in Wasser gekühlt und mit Hülfe der Wasserluftpumpe über ein Asbestfilter filtrirt. Nach dem Auswaschen mit heissem Wasser, Alkohol und $\bar{A}$ ther und Reduction im Wasserstoffstrome hatte das Filterrohr um $0,0036 \mathrm{~g}$ zugenommen. (Vgl. Jahresb. 1887 S. 939.)

Zur eigentlichen Reductionsbestimmung wurde die Kupferlösung 4 Minuten lang für sich gekocht, sodann 25 ec von der ursprünglichen Zuckerlösung

ohne Spodium 99,44 Proc. Zucker mit Spodium 99,26

Mit zunehmender Temperatur vergrössert sich der Fehler ungleich mehr, als nach der Tuchsehmid'. schen Formel. 
zugesetzt und abermals 4 Minuten im Kochen gehalten. Das Filterrohr hatte nach der Reduction eine Gewichtsvermehrung von $0,0137 \mathrm{~g}$ erfabren,

daher abzüglich der obigen $0,0036 \mathrm{~g}$ eine wirkliche Gewichtsvermehrung von $0,0101 \mathrm{Cu}$.

Wenn nach $\mathrm{B}$ odenbender $141 \mathrm{mg} \mathrm{Ca}=50 \mathrm{mg}$ Invertzucker, so würden in 50 ce Zuckerlösung

$$
\frac{50.0,0101}{141}=0,0035 \mathrm{~g} \text {, in } 100 \mathrm{cc} \text { also }
$$

$0,0140 \mathrm{~g}$ Invertzucker vorhanden sein.

Da die Menge der Zuckerlösung für die weitere Untersuchung leider nicht langte, so musste die Prüfung der Invertzuckervermehrung nach der Behandlung mit Spodium in einer zweiten Probe vorgenommen werden.

Angewendet: $26,048 \mathrm{~g}$ Zucker zu $200 \mathrm{cc}$ gelöst. Directe Polarisation $98,56^{\circ}$ $100 \mathrm{cc}$ der Lösung wurden mit $10 \mathrm{~g}$ Spodiumgries unter kräftigem Umschütteln 2 Minuten behandelt. Die Polarisation der Lösung betrug sodann $93,4^{\circ}$. $25 \mathrm{cc}$ dieser Lösung, wie oben beschrieben, mit $100 \mathrm{cc}$ Soldaini'schem Reagens gekocht, ergab nach der Reduction im Wasserstoffstrome eine Gewichtsvermehrung des Filterrohrs um $0,0168 \mathrm{~g}$.

Nach Abzug der $0,0036 \mathrm{~g} \mathrm{Cu}$ aus dem Soldainischen Reagens würde sich also eine reducirte Kupfermenge von ergeben, einer Invertzuckermenge von 0,0048 oder in $100 \mathrm{cc}$ Lösung von entsprechend.

$0,143 \mathrm{~g}$

In Berücksichtigung der verschiedenen Mengen Trockensubstanz in den beiden verwendeten Zuckerproben $(97,67: 98,56)$ würde dies auf den ersten Zucker berechnet einer Menge von $0,1112 \mathrm{~g}$ Invertzucker entsprechen. Die mit Knochenkohle behandelte Probe hätte somit eine Vermehrung von 0,0318 Proc. Invertzucker erfahren.

Ist die Drehung des Invertzuckers nach Gubbe

$$
\begin{gathered}
\text { für } 0,-30^{\circ} \mathrm{C} \cdot \alpha_{D}^{t}=-23,305 \\
+0,30406(t-20)+0,001654(t-20)^{2} \\
\text { für } t^{22} \text { also } \frac{0,0318-23,92}{100}=-0,0076^{0}
\end{gathered}
$$

oder für Ventzke-Soleil unter Anwendung des $200 \mathrm{~mm}$ Rohrs, wenn $1^{0}$ Ventzke $=0,345$ Kreistheilung, würde die Lösung nach der Behandlung mit Knochenkohle eine Verminderung der Drehung um $0,04^{\circ}$ erfahren haben. Aber selbst angenommen, dass die Vermehrung des Invertzuckers keine scheinbare, d. h. innerhalb der Grenzen der Versuchsfebler liegende ist, sondern thatsächlich stattfand, würde die hierdurch bewirkte Minusdrehung so unbedeutend sein, dass dieselbe mit dem Farbenapparat und der Zehntelnoniusskala gar nicht zu ermitteln wäre.

II. Absorption in Osmosewässer-und Melasselösungen. Es erübrigt noch zu prüfen, ob und wieweit die Verhältnisse der Zuckerabsorption in Melasse und Osmosewasserlösungen von jenen in reinen Zuckerlösungen abweichen.

Es wurde derart verfahren, dass auf die zu 100 cc gelöste Substanz 10 cc Bleiacetat gesetzt und $50 \mathrm{cc}$ des Filtrates mit $5 \mathrm{~g}$ pulverisirter Knochenkohle behandelt und nach

\begin{tabular}{|c|c|c|c|c|}
\hline \multirow[b]{2}{*}{ Angewendet: } & \multicolumn{2}{|c|}{ Polarisation } & \multirow{2}{*}{$\begin{array}{l}\text { Ange- } \\
\text { wendeter } \\
\text { Zucker } \\
\text { in }\end{array}$} & \multirow{2}{*}{$\begin{array}{c}\text { Dav, ab- } \\
\text { sorbirt } \\
\text { Proc. }\end{array}$} \\
\hline & $\begin{array}{l}\text { ohne } \\
\text { Spod. }\end{array}$ & $\begin{array}{c}\text { mit } \\
\text { Spod. }\end{array}$ & & \\
\hline 14,465 & 27,05 & 24,87 & 3,905 & 8,06 \\
\hline II. 16,236 & 37,587 & 36,10 & 6,101 & 3,93 \\
\hline III. 16,860 & 29,60 & 28,1 & 4.990 & 5,06 \\
\hline IV 18.212 & 3249 & 30.58 & 591 & 5,87 \\
\hline
\end{tabular}
2 Minuten filtrirt wurde. Die Ergebnisse bei vier verschiedenen Osmosewässern sind aus folgender Zusammenstellung ersichtlich:

Wie ersichtlich, ist eine gleichmässige Ein wirkung nicht wahrnehmbar. Bei II und IV kamen ziemlich gleiche Zuckermengen in Verwendung und die Differenz in der Absorption betrug 1,94 Proc. des Zuckers.

Einfluss der Bleizuckerlösung auf die Absorption. Da das Osmosewasser IV von ziemlich heller Farbe war, konnte noch eine weitere Prüfung vorgenommen werden, ob die Gegenwart der Bleizuckerlösung die Absorption beeinflusst. Zur Beobachtung musste jedoch das $100 \mathrm{~mm}$ Rohr verwendet werden.

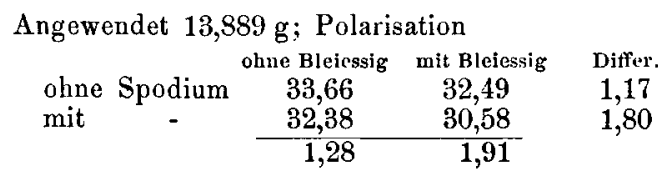

Aus der mit basisch essigsaurem Blei gereinigten Lösung wurde also thatsächlich mehr Zucker absorbirt, da trotz der geringeren Zuckermenge bei letzterer Probe die procentische Absorption entgegen den durch Walberg beobachteten umgekehrten Verhältnissen eine mindere war.

Bei Melasse gestalteten sich die $\mathrm{Ab}-$ sorptionsverhältnisse folgendermassen:

$\begin{array}{cccccc}\text { Angewendet } & \begin{array}{c}\text { Polar. } \\ \text { ohne Spod. }\end{array} & \begin{array}{c}\text { mit } \\ \text { Spodium }\end{array} & \begin{array}{c}\text { Angewand- } \\ \text { ter Zucker } \\ \text { g }\end{array} & \begin{array}{c}\text { davon } \\ \text { abrorbirt } \\ \text { Proc. }\end{array} \\ \text { I. } & 13,028 & 48,46 & 45,43 & 6,31 & 6,25 \\ \text { II. } & 13,028 & 50,27 & 46,68 & 6,54 & 7,12\end{array}$

So weit aus der geringen Anzahl Untersuchungen ein Vergleich hier zulässig, würde sich ergeben, dass die Absorption in $\mathrm{Me}-$ lasselösungen bedeutender ist, als bei Osmosewässern.

Absorption bei Gegenwartvon Essigsäure. Der Umstand, dass in salzsaurer Lösung die Absorption des Zuckers eine verschwindend geringe war gegen jene in neutraler oder basischer Lösung, liess vermuthen, dass die Eigenschaft der $\mathrm{Ab}$ sorptionsverminderung oder Verhinderung auch durch andere, nicht invertirende Säuren 
bewirkt werden könne. Es wäre damit ein Weg angezeigt, den durch unvermeidliche Anwendung der Knochenkohle entstehenden Fehler auszugleichen. Die nachfolgenden Versuche unter Anwendung von Essigsäure beweisen, dass die Voraussetzung richtig war.

Der Versuch wurde zuerst auf reinen Zucker erstreckt.

Angewendet $13,024 \mathrm{~g}$ zu 100 ce gelóst.

Directe Polarisation ohne Spodium

Mit $5 \mathrm{~g}$ Spodium pulv. und 2 Min. Dauer unter kräftigem Schütteln Differenz $\frac{93,8}{6,12}$

Eine zweite Probe von abermals $13,024 \mathrm{~g}$ des Zuckers wurde zu 100 cc gelöst, jedoch unter Zusatz von 25 ce Essigsäure von 1,0513 spec. Gew.

Directe Polarisation $99,82^{\circ}$

Mit $5 \mathrm{~g}$ Spodium pulv. auf 50 cc Lösung u. 2 Min. Dauer wie oben.

$$
\text { Polarisation } \frac{99,10}{0,72}
$$

Es fañd also thatsächlich eine ungemein verminderte Absorption statt, da bei derselben Kohle und denselben Umständen und ohne Beigabe von Essigsäure etwa 6,12 Proc. absorbirt wurden.

Da bei Melassen und Osmosewässern die Absorption überhaupt geringer, so konnte möglicherweise durch Anwendung von Essigsäure dieselbe auf ein Minimum herabgedrückt werden.

Versuch mit Melasse:

I. Angewendet: $13,024 \mathrm{~g}$ mit 10 ce Bleiacetat zu 110 cc gelöst.

Directe Polarisation

$47,96^{\circ}$

Mit $5 \mathrm{~g}$ Spodium wie oben

Differenz $\frac{43,03}{4,93}$

Weiterhin wurden $26,048 \mathrm{~g}$ unter Zusatz von $20 \mathrm{ce}$ Bleilösung zu $110 \mathrm{cc}$ gelóst. Vom Filtrat $50 \mathrm{cc}$ mit 25 ce der Essigsãure versetzt und auf $100 \mathrm{cc}$ ergänzt.

\section{Polarisation: $\quad 47,87$ gegen 47,96 .}

Mit $5 \mathrm{~g}$ Spodium auf 50 cc derselben Lösung; die Entfärbung war vollständig.

$$
\begin{gathered}
\text { Polarisation: } \\
\text { Differenz }
\end{gathered} \frac{47,82}{0,04}
$$

II. Melasse

Genau wie oben vorgegangen.

Polarisation ohne Essigsäure u. ohne Spodium 53,25

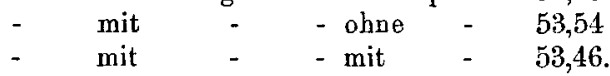

III. Melasse

Polarisation ohne Essigsäure u. ohne Spodium 50,6

$$
\begin{aligned}
& \text { - mit - } \quad \text { - } \quad \text { - } 51,17 \\
& \text { - mit - } \quad \text { - mit } \quad \text { - } 50,66 \text {. }
\end{aligned}
$$

I. Osmosewasser

Polarisation ohne Essigsäure u. ohne Spodium 32,62

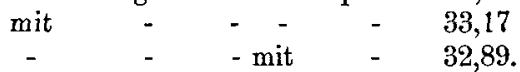

II. Osmosewasser

Polarisation ohne Essigsäure u. obne Spodium 32,78

$$
\begin{array}{ccccccc}
- & \text { mit } & - & - & - & - & 34,36 \\
& \cdot & - & - \text { mit } & - & 33,44
\end{array}
$$

III. Osmosewasser

Polarisation ohne Essigsäure u. ohne Spodium 32,67 mit $\quad$ - $\quad$ - $\quad$ - $\quad-\quad 33,00$

- mit - 33,28 .

Während also bei Melasse die erhaltenen Resultate recht befriedigend waren, lassen dieselben bei Osmosewässern noch zu wünschen übrig. Hier ist sogar bei Probe II und III die auffallende Frscheinung zu bemerken, dass die Polarisation nach der Behandlung mit Essigsäure und Knochenkohle eine böhere war. Offenbar kann der Grund hierfür in der vermehrten Anwesenheit von Stoffen gesucht werden, welche, wie Asparagin, Asparaginsäure und Glutin in saurer Lösung eine Pluspolarisation annehmen. Bei Probe III dürfte ausserdem die Absorption eines linksdrehenden Stoffes die Vermehrung der Drehung bewirkt haben. Kann also auch der vorgeschlagene Weg angesichts der Fehler und der Ungleichheit der Stoffe keineswegs als völlig genau gelten, so dürfte sich derselbe doch angesichts der mit $\mathrm{Me-}$ Jasse erbaltenen Resultate einer weiteren Prüfung empfehlen.

Möglicherweise genügt eine geringe Änderung des Verfabrens für Producte, welche wie Osmosewässer oder Elusionslaugen mehr organischen Nichtzucker enthalten, um die Differenzen auch hier auf ein natürlich immer schwankend bleibendes Minimum herabzudrücken -- etwa durch einen vermehrten Zusatz von Bleilösung, oder verminderten Zusatz von Essigsäure.

Kurz zusammengefasst würde sich aus dieser Arbeit Folgendes ergeben:

Die procestuale Absorption des Zuckers nimmt, wie Walberg beobachtet, mit der Concentration ab.

Die Form der Knochenkohle beeinflusst die Wirkung, ebenso wie mechanische Bewegung.

Der Salzgehalt der Melasse bewirkt keine Verminderung der Absorption.

In Verhältnissen, wie sie den bei der Untersuchung von Nachprodukten angewendeten entsprechen, ist die Absorption in 20 Minuten beendet. In den ersten Minuten ist die Wirkung naturgemäss am stärksten, nimmt jedoch allmählich ab.

Eine Inversion des Zuckers findet dabei nicht oder doch nur höchst unbedeutend statt, und hat dieselbe auf die Abnahme der Polarisation keinen Einfluss.

In salzsaurer Lösung findet unbeschadet der Absorption des Farbstoffes eine verhältnissmässig ganz unbedeutende Aufuahme von Zucker statt. Es ist diese Erscheinung nicht etwa auf die Characterverschiedenheit des Invertzuckers zurückzuführen, sondern 
wahrscheinlich auf die Eigenschaft der Säure, von den Poren leichter aufgenommen zu werden, und folgedessen die Aufnahme des Zuckers zu verhindern. Die gleiche Eigenschaft der nicht invertirenden Essigsäure bekräftigt diese Ansicht.

Eine Gleichmässigkeit der Absorption bei verschiedenen Produkten ist nicht wahrnehmbar. In reiner Zuckerlösung wird mehr absorbirt als in Melasse, da wieder mehr als in Osmosewasser.

Ein constanter Factor lässt sich auch bei sorgfältiger Einhaltung derselben Bedingungen nicht in Anwendung bringen.

Die Eigenschaft der Essigsäure, die Absorption des Zuckers, jedoch nicht jene der Farbstoffe zu verhindern, kann benutzt werden, um den, durch die für Farbenapparate oft nicht zu umgehende Anwendung der Knochenkohle, entstehenden Fehler zu compensiren.

Ich schliesse diese keineswegs erschöpfende Arbeit mit der Bemerkung, dass ich mir deren Weiterverarbeitung durchaus nicht vorbehalte.

Kolin, 12. Juni 1888.

\section{Brennstoffe.}

Die Nutzbarmachung der theerartigen Rückstände, welche bei der Erdölreinigung beim Behandeln mit Schwefelsäure entstehen, geschieht nach W. P. Thom pson (J. Ch. Ind. 1888 S. 303) von der Société Oléo Graisse nach Ravé's Verfahren in folgender Weise:

Die Masse wird mit etwas mehr Eisenspänen o. dgl. zusammengeknetet als dem Schwefelgehalte entspricht, und darauf sich selbst überlassen. Wenn die Bildung von Eisensulfat beendet ist, laugt man die Mischung mit heissem Wasser aus. Das Sulfat gebt in Lösung, die überschüssigen Eisenspäne fallen zu Boden und die Theermasse schwimmt auf der Flüssigkeit. Dieselbe wird durch weiteres Auswaschen vollends gereinigt und dann durch Erhitzen in einem Behälter verdickt. Die Masse soll nun die Eigenschaften eines guten Bitumens haben und zugleich so elastisch und zähe wie Gummi sein.

Dies Product wird als "MineralKautschuk" in den Handel gebracht. $1 \mathrm{t}$ der ersten Masse soll fast $5 \mathrm{hk}$ dieses gereinigten Bitumens ergeben. Wird dasselbe weiter erhitzt, so entwickeln sich viel flüchtige Stoffe, und es bleibt eine in Naphta lösliche Masse zurück, welche nach dem Erkalten fast so hart wie Ebonit sein soll. Als ausgezeichneter Nichtleiter der Elektricität und wegen ihrer Widerstandsfähigkeit gegen Säuren und Alkalien findet dieselbe eine ausgedehnte Anwendung. Je nach der Länge des Erhitzens lassen sich verschiedene Härtegrade herstellen. Im erweichten Zustande lässt sich die Masse auch zum Ausfüllen von Formen verwenden.

Wird das Bitumen mit 40 Proc. Sägespänen gemischt, mit etwas Kalk erhitzt und gepresst, so sollen sie einen ausgezeichneten Brennstoff abgeben.

Das Bitumen ergibt, in Naphta oder andern leichten Kohlenwasserstoffen gelöst, einen schwarzen Lack, welcher an Metall sehr fest haftet und zugleich vollkommen wasserdicht ist. Schliesslich lässt sich aber auch das Bitumen durch Erhitzen in

17 Proc. leicht brennbare Öle

$\begin{array}{rll}8 & \text { schwer } & \\ 25 & \text { Kohlenstoff zerlegen. }\end{array}$

$-e$.

Lichtmessung. W. J. Dibdin (J. Ch. Ind. 1888 S. 367) theilt die Ergebnisse seiner Untersuchungen äber Lichtmessung durch Normalflammen mit. Derselbe bestätigt zunächst wieder die Unzuverlässigkeit der Normalkerzen, welche z. B. bei Erhöhung der Zimmertemperatur viel $\mathrm{zu}$ hohe Resultate ergeben.

Von den Normalflammen verwirft $D i b-$ din auch die Amylacetatlampe und empfiehlt nur die Vernon Harcourt'sche PentanLichtgasflamme, welche allein allen Anforderungen genügen soll. (Vgl. jedoch Ferd. Fischer: Chemische Technologie der Brennstoffe S. 73 u. 342 .)

\section{Apparate.}

Messgefäss. Zum schnellen und genauen Messen bestimmter Theile von Flüssigkeiten, z. B. von Salpetersäure zum Lösen von Stahl für Eggert's colorimetrisches Verfahren, verwendet P. Vanier (J. Anal. 1888 S. 144) die in Fig. 149 dargestellte Messbürette. Behälter $a$ enthält die Flüssigkeit, welche vermittels des Dreiweghahns $d$ in den Messcylinder $b$ gelassen wird, bis sie aus der 1 bis $1,5 \mathrm{~mm}$ weiten Öffnung $o$ in den Behälter $c$ überfliesst. Man schliesst nun den Zufluss $a$ ab und lässt Rohr $b$ nach $h$ hin ausfliessen, wobei die in der Capillarröhre $f h$ etwa verbleibenden Tropfen unberücksichtigt bleiben. Sobald sich in $e$ eine grössere Menge Flüssigkeit gesammelt hat, lässt man diese durch den Hahn $e$ ausfliessen, 